Supporting Information

\title{
Dinitrile-Mononitrile Based Electrolyte System for Lithium-Ion Battery Application with the Mechanism of Reductive Decomposition of Mononitriles
}

Rupesh Rohan, ${ }^{\dagger}$ Tsung-Chieh Kuo, ${ }^{\dagger}$ Jing-Heng Lin, ${ }^{+} \mathrm{Ya}-\mathrm{Chu} \mathrm{Hsu},{ }^{+}$Chia-Chen Li, ${ }^{\ddagger} \mathrm{Jyh}-\mathrm{Tsung}$

$L e e^{*+s}$

†Department of Chemistry, National Sun Yat-sen University, Kaohsiung 80424, Taiwan.

$\ddagger$ Department of Material Science and Resource, National Taipei University of Technology, Taipei 10608, Taiwan.

${ }^{\S}$ Department of Medicinal and Applied Chemistry, Kaohsiung Medical University, Kaohsiung 80708, Taiwan.

\section{Corresponding Author}

*Email: jtlee@faculty.nsysu.edu.tw; tel: +886-7-525-3951. 


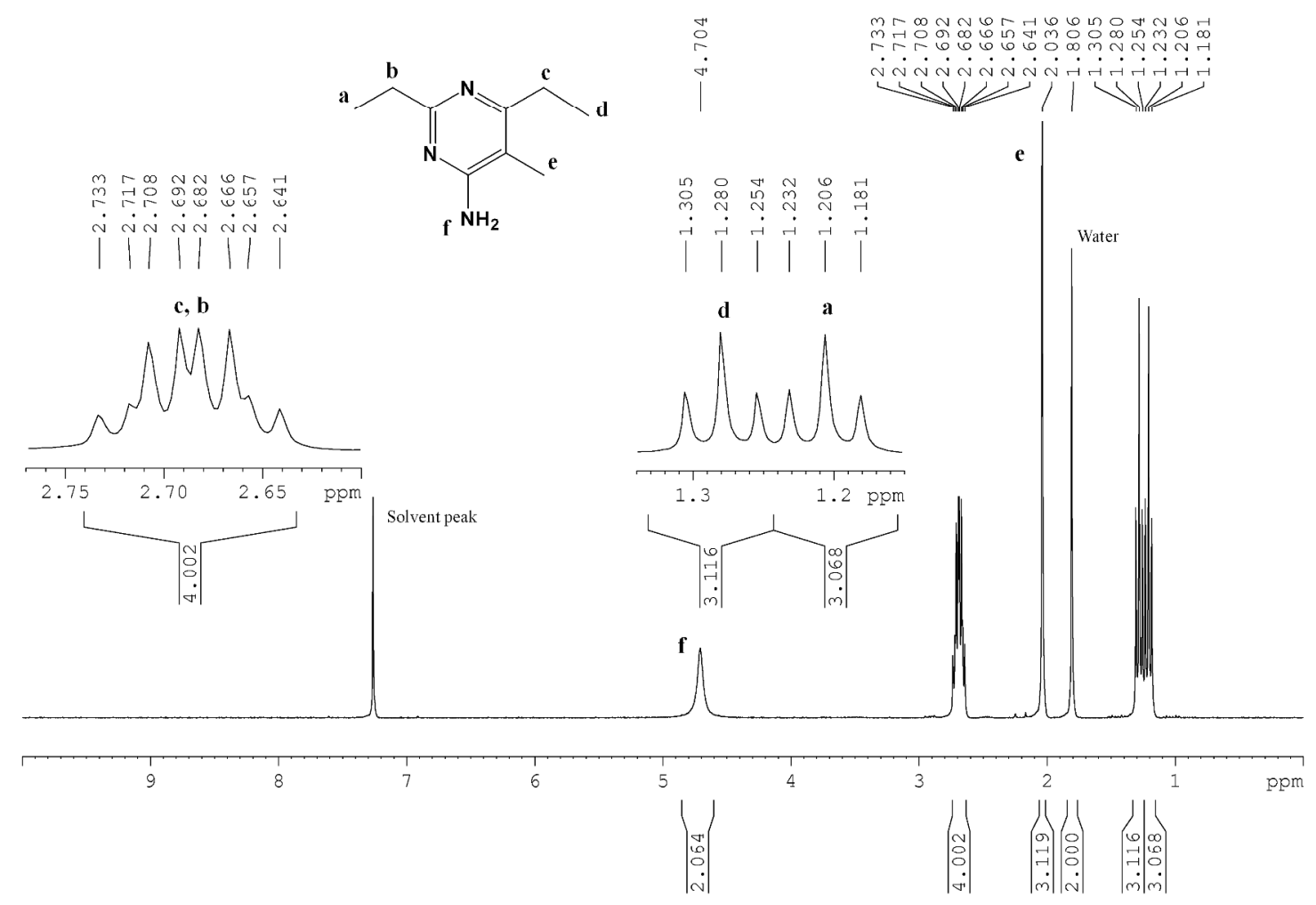

Figure S1. ${ }^{1} \mathrm{H}$ NMR spectrum of 2,6-diethyl-5-methylpyrimidin-4-amine, a trimer formed from propoinitirle decomposition. 


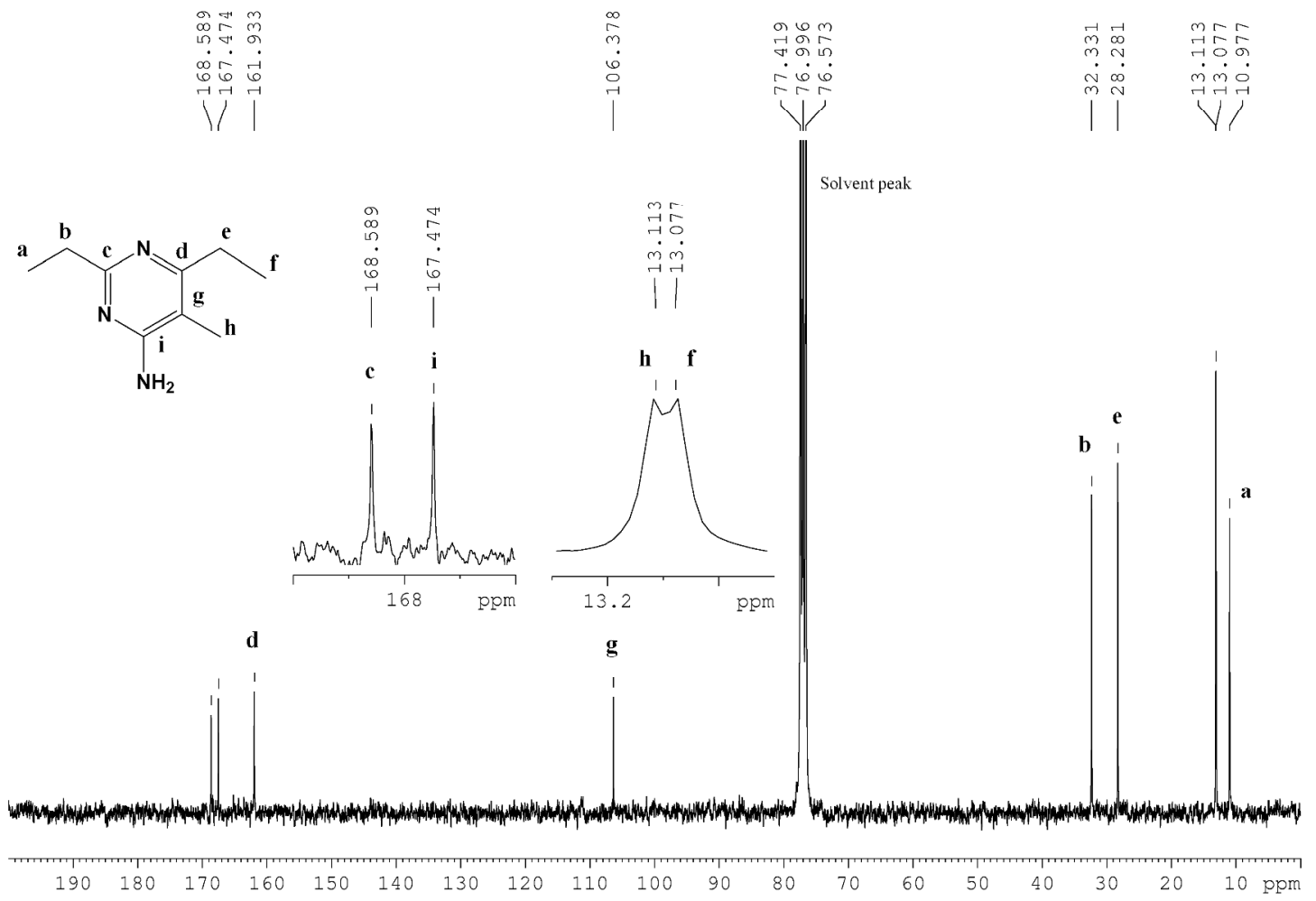

Figure S2. ${ }^{13}$ C NMR spectrum of 2,6-diethyl-5-methylpyrimidin-4-amine, a trimer formed from propoinitirle decomposition. 


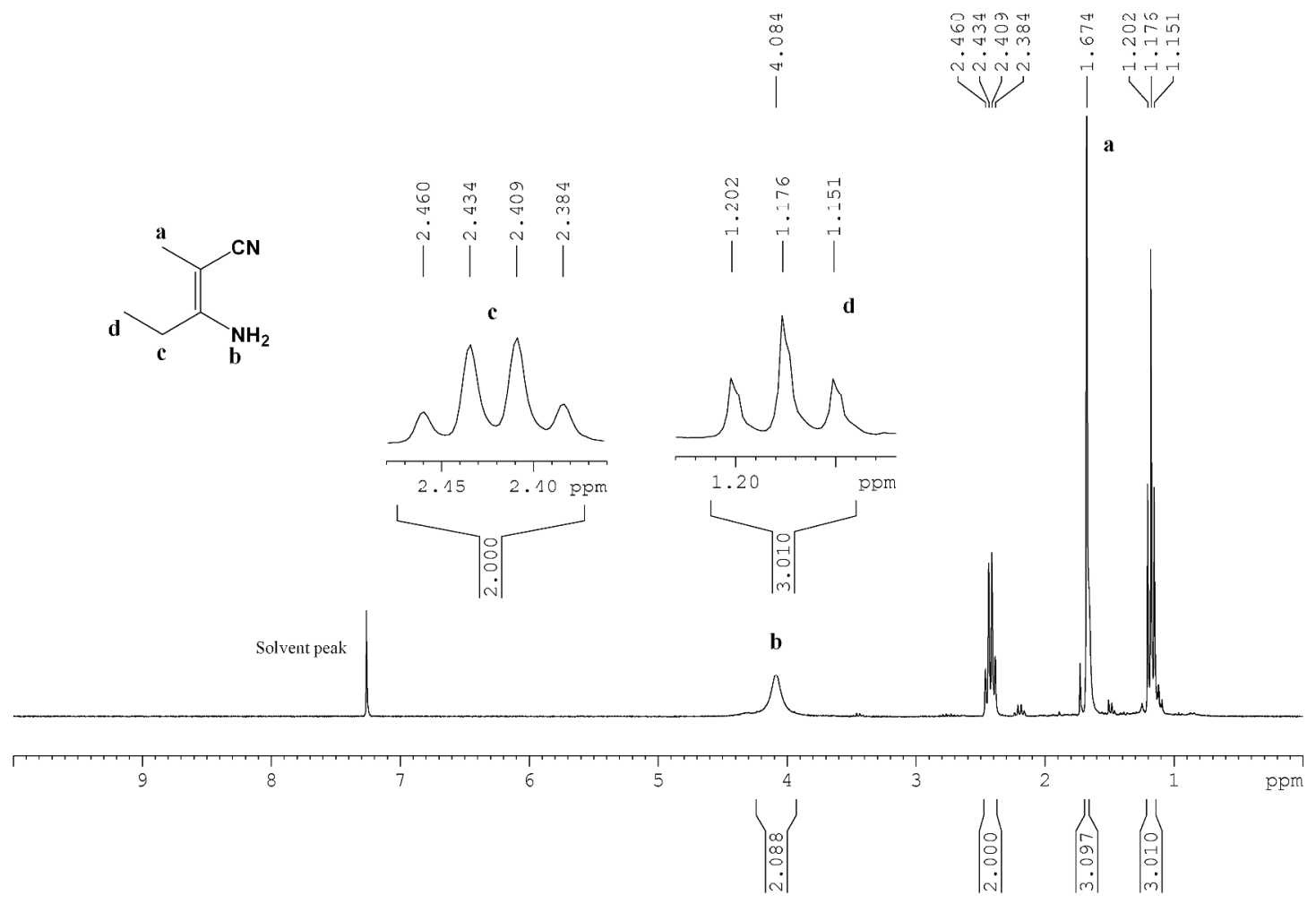

Figure S3. ${ }^{1} \mathrm{H}$ NMR spectrum of 3-amino-2-methylpent-2-enenitrile, a dimer formed from propoinitirle decomposition. 


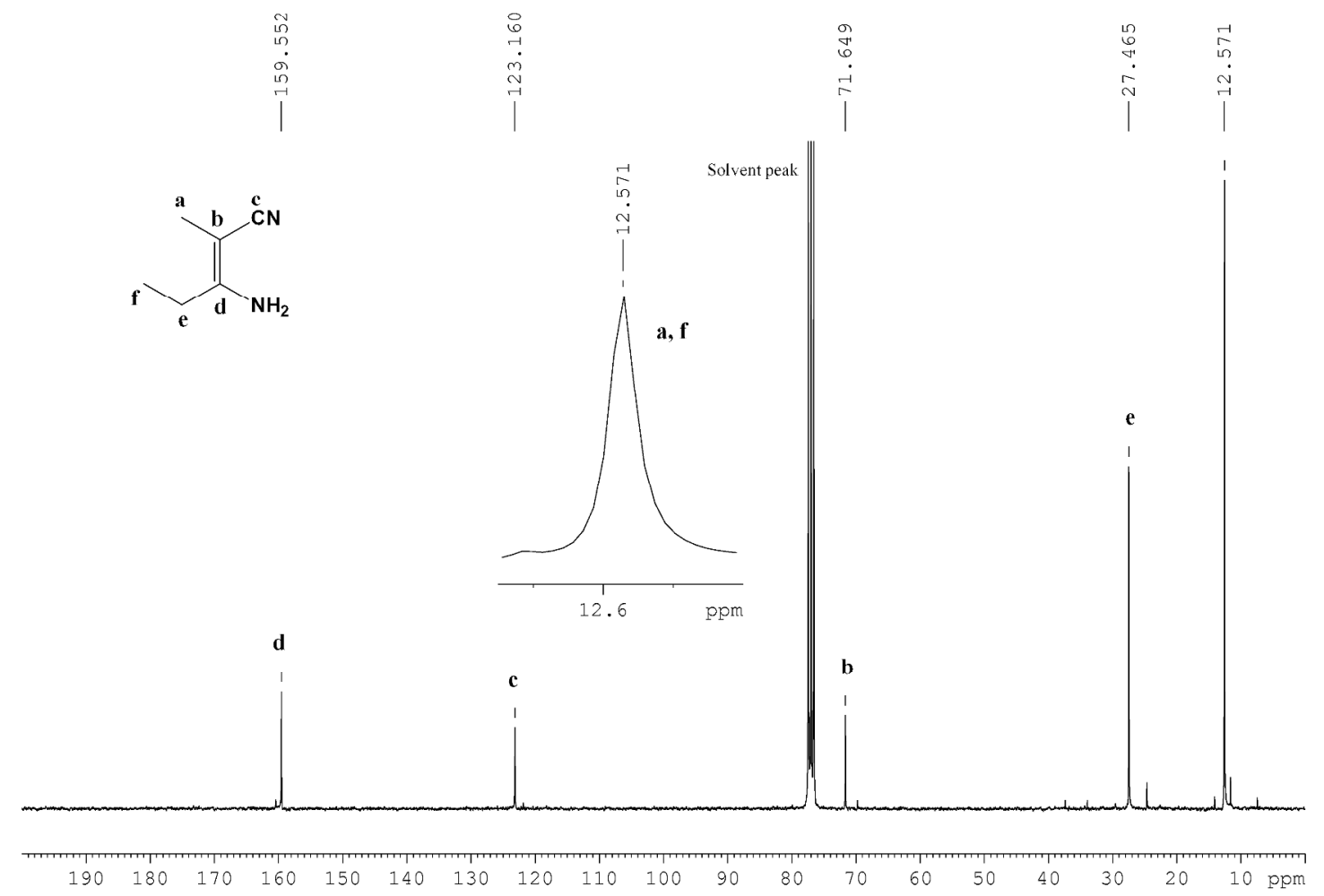

Figure S4. ${ }^{13} \mathrm{C}$ NMR spectrum of 3-amino-2-methylpent-2-enenitrile, a dimer formed from propoinitirle decomposition. 


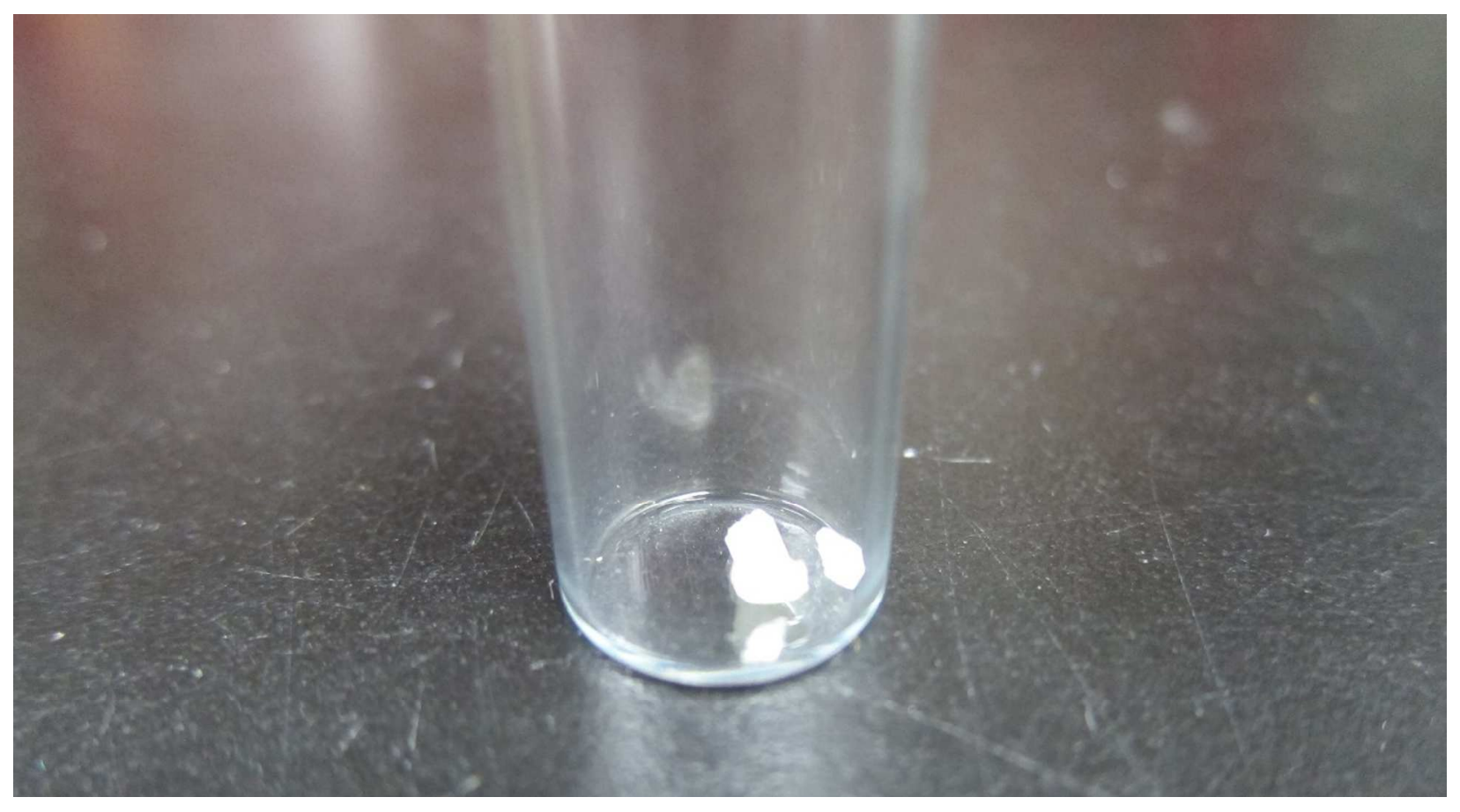

Figure S5. Colorless purified crystals of 2,6-diethyl-5-methylpyrimidin-4-amine, a trimer formed from propoinitirle decomposition. 


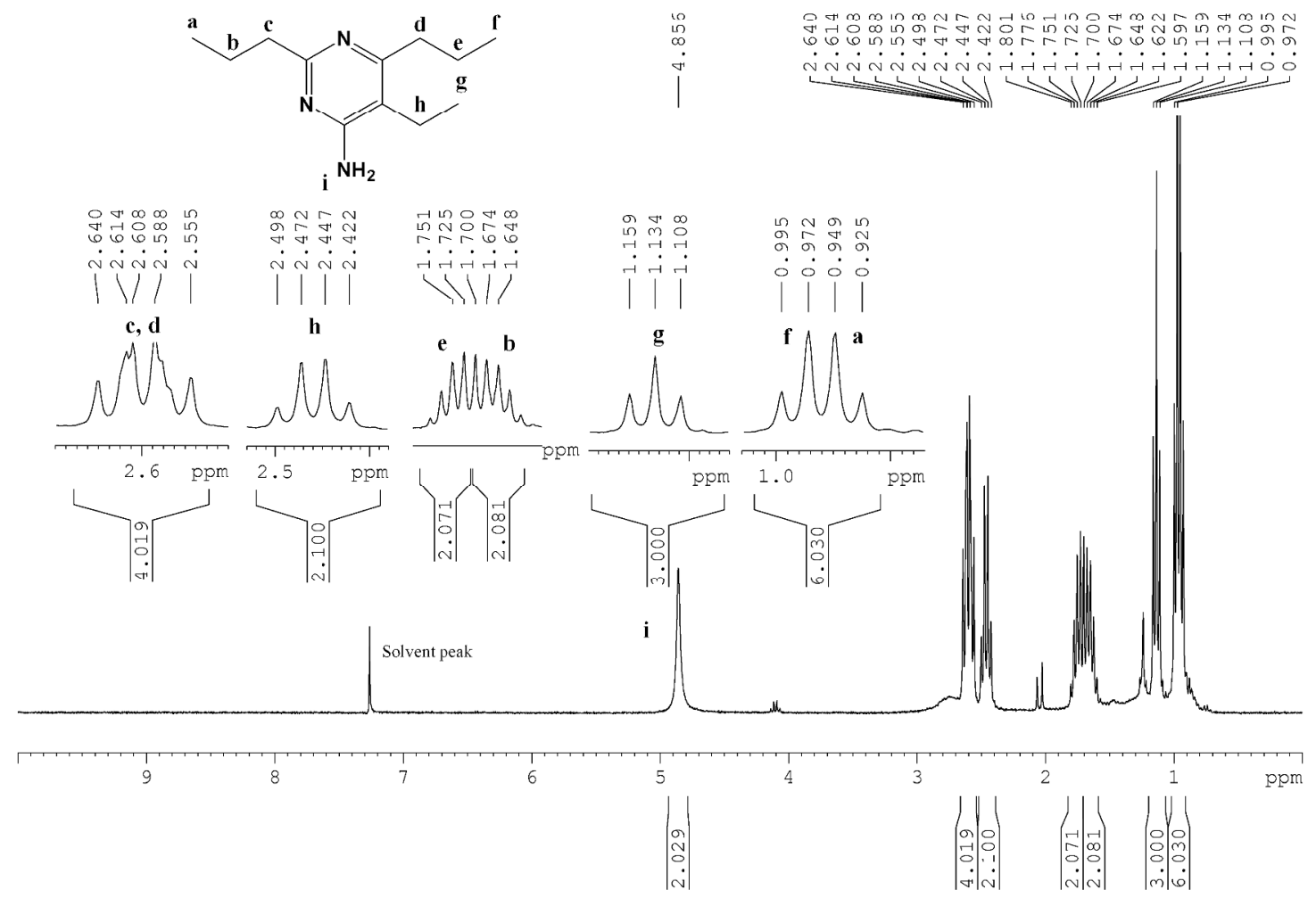

Figure S6. ${ }^{1} \mathrm{H}$ NMR spectrum of 2,6-dipropyl-5-ethylpyrimidin-4-amine, a trimer formed from butyronitrile decomposition. 


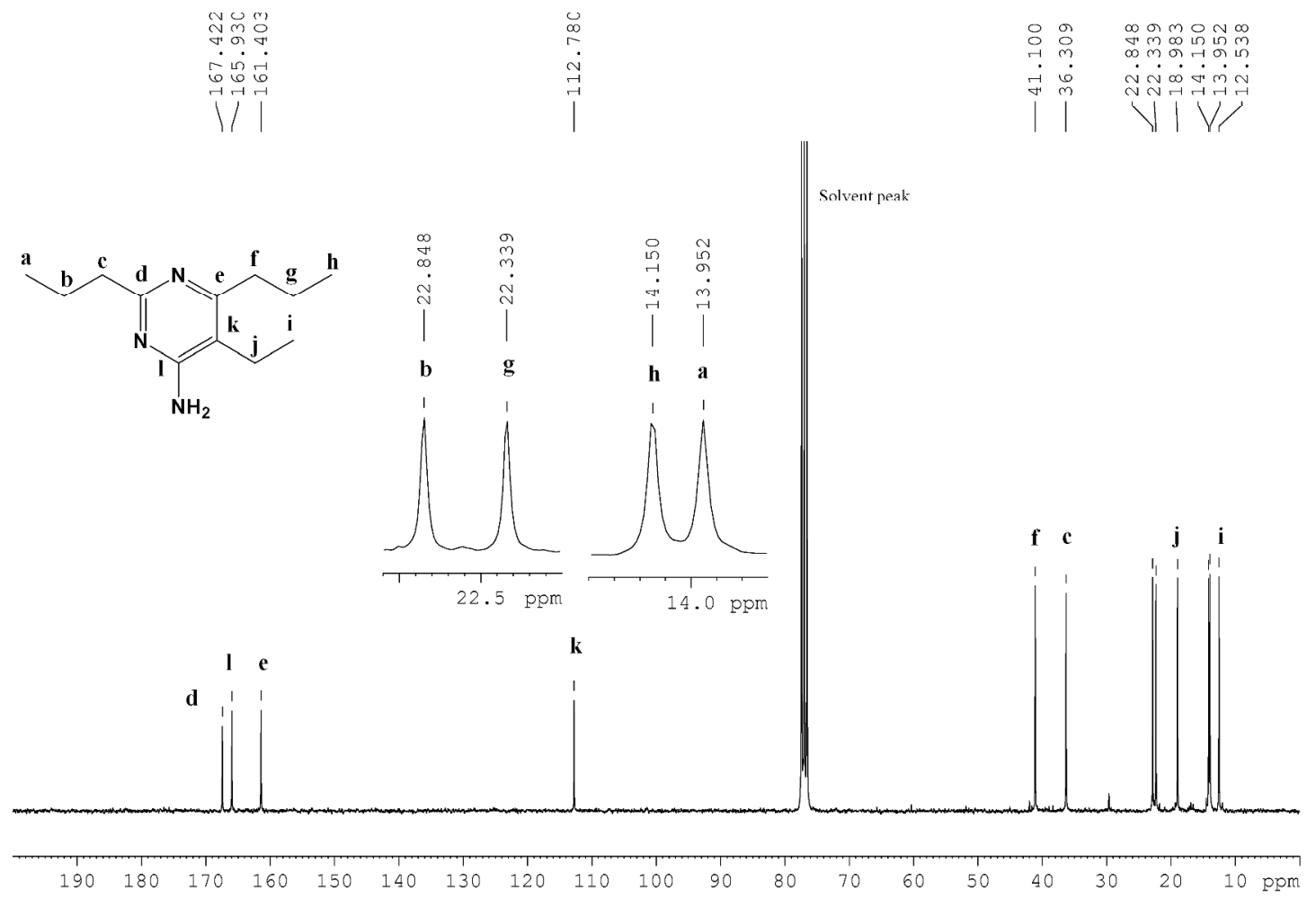

Figure S7. ${ }^{13} \mathrm{C}$ NMR spectrum of 2,6-dipropyl-5-ethylpyrimidin-4-amine, a trimer formed from butyronitrile decomposition. 

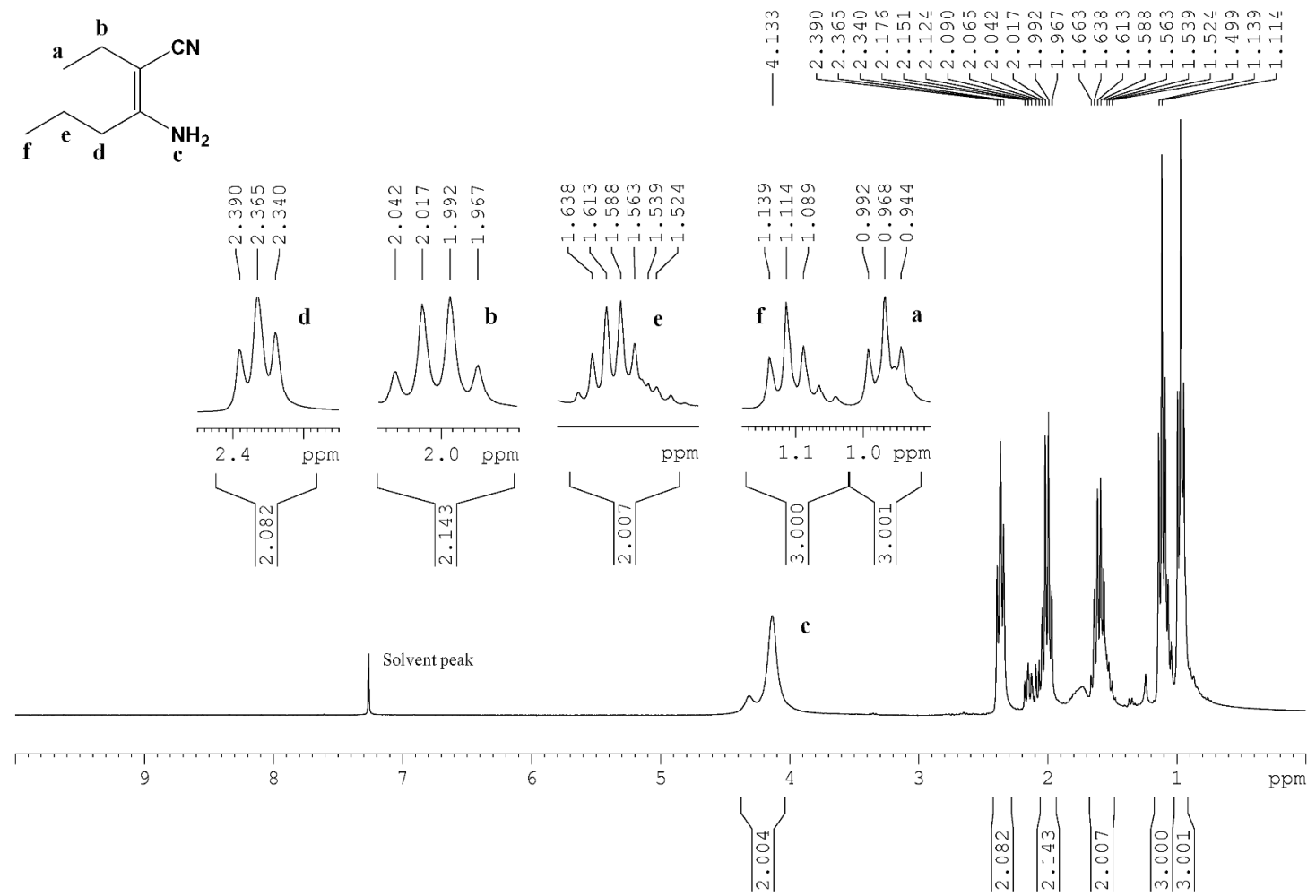

Figure S8. ${ }^{1} \mathrm{H}$ NMR spectrum of 3-amino-2-ethylhex-2-enenitrile, a dimer formed from butyronitrile decomposition. 


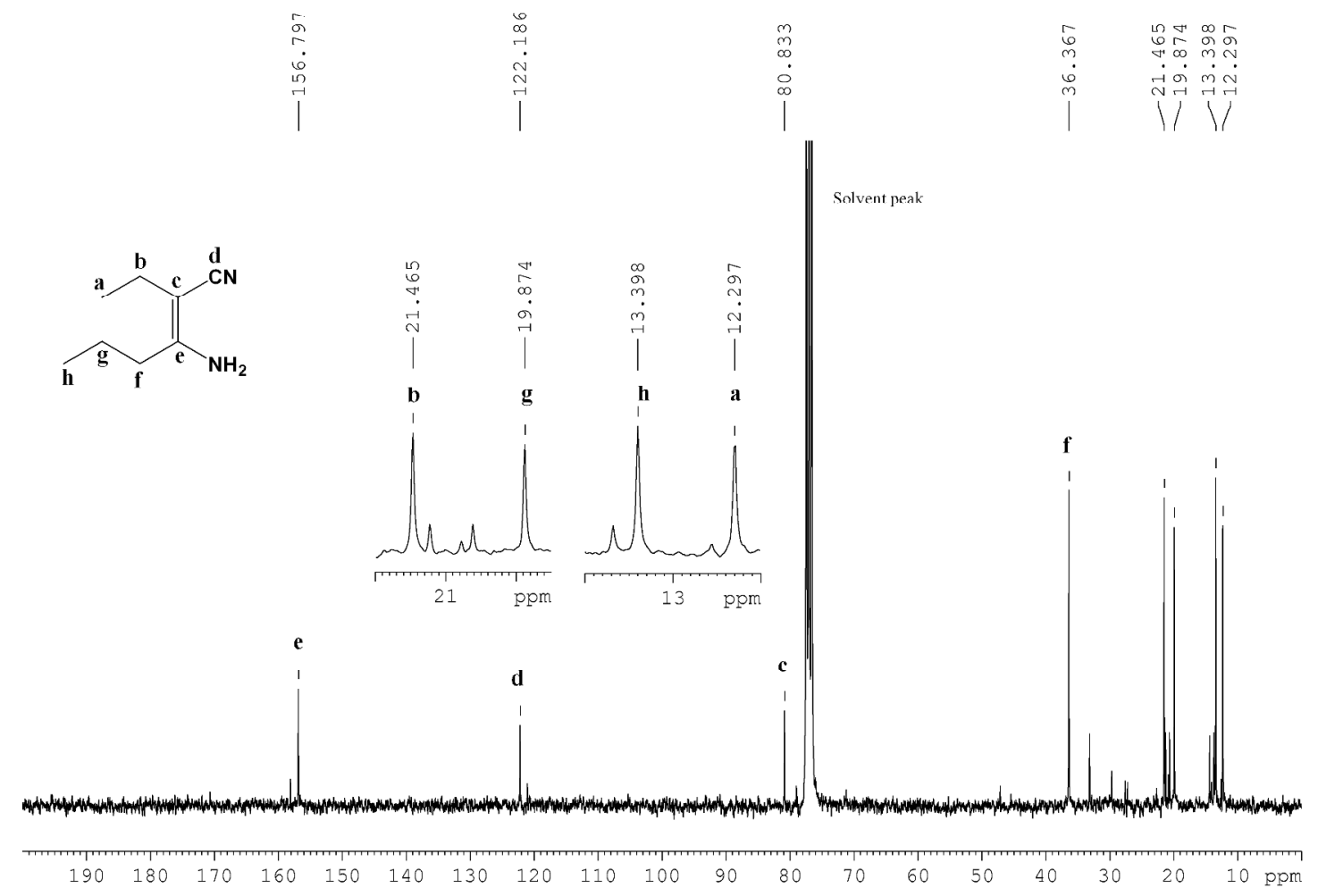

Figure S9. ${ }^{13} \mathrm{C}$ NMR spectrum of 3-amino-2-ethylhex-2-enenitrile, a dimer formed from butyronitrile decomposition. 


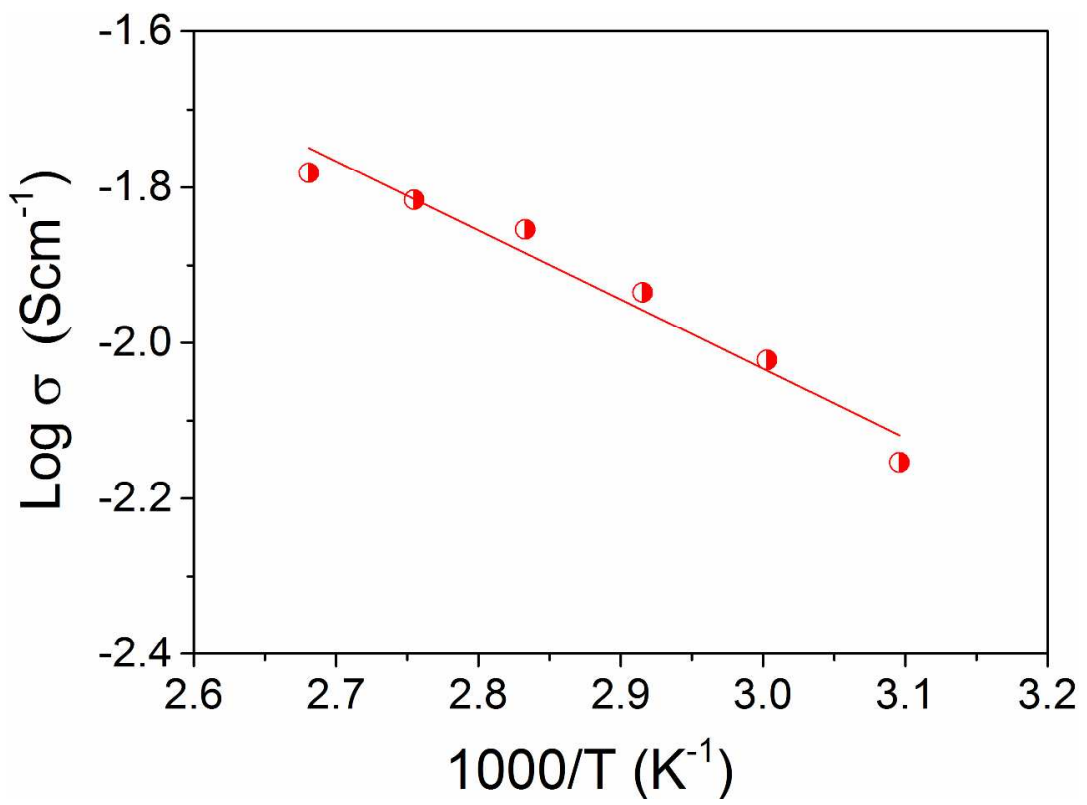

Figure S10. Arrhenius plot of 1.0 M LiTFSI in glutaronitrile/butyronitrile (6/4) with 5\% VC electrolyte. 

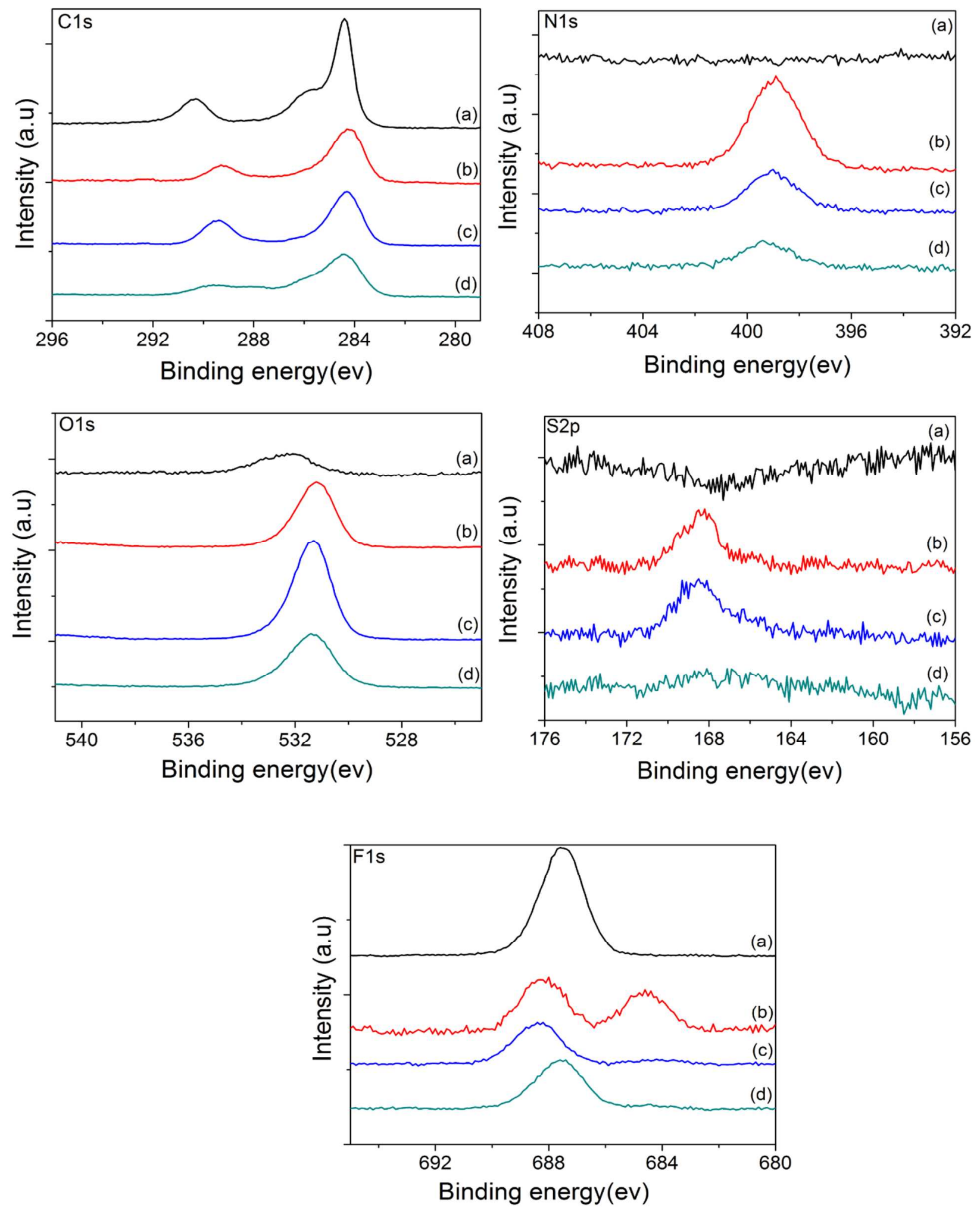

Figure S11. C 1s, N 1s, O 1s, S 2p, and F 1s XPS spectra for (a) pristine MCMB electrode and MCMB electrodes in $1.0 \mathrm{M}$ LiTFSI in glutaronitrile/butyronitrile (6/4) with (b) $0 \%$, (c) $2 \%$, and (d) $5 \%$ VC electrolytes after $4 \mathrm{CV}$-cycles at $30{ }^{\circ} \mathrm{C}$. 
Table S1. Surface chemical composition of XPS analysis for pristine MCMB electrode and MCMB electrodes in 1.0 M LiTFSI in glutaronitrile/butyronitrile (6/4) with 0\%, 2\%, and 5\% VC electrolytes after $4 \mathrm{CV}$-cycles at $30^{\circ} \mathrm{C}$.

\begin{tabular}{cccccc}
\hline VC addition & \multicolumn{5}{c}{ Composition (atomic concentration, \%) } \\
\cline { 2 - 6 }$($ Wt \% $)$ & C 1s & N 1s & O 1s & F 1s & S 2p \\
\hline Pristine MCMB & 72.96 & 0.0 & 2.41 & 24.64 & 0.0 \\
$\mathbf{0}$ & 55.97 & 5.24 & 35.19 & 2.97 & 0.62 \\
$\mathbf{2}$ & 49.88 & 2.25 & 44.58 & 2.58 & 0.71 \\
$\mathbf{5}$ & 57.49 & 1.44 & 34.37 & 6.51 & 0.19 \\
\hline
\end{tabular}

